\title{
Itinéraires
}

Itinéraires Littérature, textes, cultures

2010-2 | 2010

Les blogs

\section{Copier-coller Baudelaire : fonctions de la citation littéraire sur les blogs}

\section{Mathilde Labbé}

\section{(2) OpenEdition}

1 Journals

\section{Édition électronique}

URL : http://journals.openedition.org/itineraires/2057

DOI : $10.4000 /$ itineraires.2057

ISSN : 2427-920X

Éditeur

Pléiade

\section{Édition imprimée}

Date de publication : 1 juillet 2010

Pagination : 147-153

ISBN : 978-2-296-12012-9

ISSN : 2100-1340

Référence électronique

Mathilde Labbé, « Copier-coller Baudelaire : fonctions de la citation littéraire sur les blogs », Itinéraires [En ligne], 2010-2 | 2010, mis en ligne le 01 juillet 2010, consulté le 20 avril 2019. URL : http:// journals.openedition.org/itineraires/2057; DOI : 10.4000/itineraires.2057

\section{(ब) $(\Theta \Theta$}

Itinéraires est mis à disposition selon les termes de la licence Creative Commons Attribution - Pas d'Utilisation Commerciale - Pas de Modification 4.0 International. 


\title{
Copier-coller Baudelaire : fonctions de la citation littéraire sur les blogs
}

\begin{abstract}
The poems and prose work of Charles Baudelaire are freely accessible on the Internet. However, despite their availability, many bloggers choose to copy-paste Baudelaire's work directly to their blogs. In this article, I consider several explanations for this phenomenon and discuss how the practice of copy-pasting lends to the construction of one's virtual identity.
\end{abstract}

Keywords : quotation, blogs, Charles Baudelaire, transmission of literary heritage, literary culture

Mots clés : citation, blogs, Charles Baudelaire, transmission du patrimoine littéraire, culture littéraire

L'usage du blog engage-t-il une transformation des pratiques d'écriture? Invasion du discours par l'intime, interactivité et construction collective de savoirs, ces trois caractéristiques communément admises de l'écriture en réseau ont-elles donné naissance à une nouvelle approche du littéraire? Il faudrait, pour répondre à ces questions, cerner des pratiques d'écriture favorisées par les blogs et qui font apparaitre leur spécificité éditoriale. Nous nous intéresserons ici à la citation littéraire, pratique à la limite de l'écriture, et qui est à la fois plus simple et moins évidemment utile dans le cadre d'un blog que dans celui d'une publication imprimée. Au cours d'une étude sur la réception de l'œuvre de Baudelaire, nous avons en effet trouvé de nombreuses pages de blogs citant in extenso des poèmes, avec ou sans introduction, alors que très peu de pages utilisent un lien hypertexte vers un site de référence. Laissant de côté le commentaire littéraire académique, qui reste rare sur les blogs, et où le collage du texte paraît secondaire, nous nous intéresserons à des articles dont la valeur, voire l'existence, semble tenir à la présence du texte copié. Proche du journal de collectionneur, du 
recueil de poèmes choisis ou du «scrapbook ${ }^{1}$ ", cette pratique de la copie semble paradoxale sur un médium d'accès public où elle apparaît comme évidemment redondante. L'œuvre étant en grande partie déjà numérisée et accessible sur des sites spécialisés ${ }^{2}$, la citation des poèmes se réduit parfois à l'exécution d'un « copier-coller ». Comment la copie s'inscrit-elle dans les pratiques d'écriture en réseau? Quelle est la fonction de cette redondance consciente qui, bien souvent, ne donne lieu ni à la réécriture, ni au commentaire? Est-ce, comme il le semble au premier abord, une copie sans usage, ou bien une promotion de la citation au rang de genre littéraire? Emmanuël Souchier a montré l'intérêt d'une étude de l' "énonciation éditoriale ${ }^{3}$ » propre à chaque médium. Or les éléments de cette énonciation ont d'autant plus de poids dans le cas qui nous intéresse, puisque celui qui édite le texte n'en est pas ici l'auteur et que le contenu du texte est déjà connu. Que l'internaute commente ou non ce don du texte ${ }^{4}$, les contraintes éditoriales liées aux plates-formes d'hébergement bâtissent un environnement graphique et textuel avec lequel le poème collé entre en résonance. Les listes adjacentes d'articles, de blogs recommandés, le «profil » affiché, les «tags » utilisés ou les commentaires de lecteurs constituent un paratexte propre au médium et porteur de sens. L'internaute recevant le poème posté perçoit en même temps, de manière diffuse, le sens que lui a donné le rédacteur du blog. L'étude de soixante blogs mentionnant Charles Baudelaire ou son œuvre a permis de dégager pour cette pratique du collage trois fonctions qui, pour être non exclusives, correspondent à trois usages relativement distincts. Tentative de transmission d'une culture patrimoniale, technique de présentation de soi ou appel à l'adhésion émotive du lecteur, le copier-coller du poème fait du texte classique un outil au service de la relation que l'auteur du blog veut créer avec son lecteur.

\section{La transmission culturelle signée par le médiateur}

La copie ou le collage du poème se présente volontiers comme un acte de transmission du patrimoine littéraire, dont la réception est supposée éducative ou divertissante. Or certains consacrent une part importante de leurs

1. Le scrapbook désigne une pratique d'édition manuelle faite de collages, mais il semble que son principe décrive bien les pratiques de navigation et d'édition sur Internet, puisque Firefox propose depuis le 11 août 2009 une extension portant ce nom et dédiée à l'organisation de pages web favorites.

2. Voir Gallica, http://www.gallica.bnf.fr ou Baudelaire Litteratura, http://baudelaire. litteratura.com par exemple.

3. Emmanuël Souchier, «L'énonciation éditoriale dans les écrits d'écran », Communication \& Langages, $\mathrm{n}^{\circ} 145$, septembre 2005, p. 6.

4. Jean Davallon a montré, dans Le Don du patrimoine (Paris, Hermès science/Lavoisier, 2006), que la transmission patrimoniale s'expliquait par la logique du don. Le collage reprend également de manière ironique le motif poétique du don du poème, puisque le copiste n'est pas l'auteur. 
articles à la mise en ligne de textes classiques, et cette tendance à l'anthologie parfois compulsive suggère que le rédacteur du blog trouve lui-même un intérêt à cette pratique. En effet, puisqu'il existe sur le réseau des versions numérisées et gratuites de l'œuvre, la citation multiple tire sa valeur de la sélection effectuée, et tient à la fois du recueil de poèmes choisis (pratique d'édition tournée vers un public) et de la collection (pratique d'édition tournée vers le plaisir personnel). Cette ambiguité est parfois sensible dans la manière dont sont introduits les poèmes : quand l'âge du rédacteur favorise l'association de Baudelaire à une lecture scolaire, l'acte de transmission du patrimoine donne lieu à une négociation entre le souhait de faire lire un texte qui risque de rebuter le lecteur jeune et l'envie plus impérieuse de le copier pour le plaisir de le relire soi-même, ou de le placer en regard d'un écrit personnel. Sur son blog, Khazard rédige des billets d'humeur auxquels il associe des poèmes :

Cher lecteur, [...] un peu de poésie pour aujourd'hui, ça ne te dérange pas j'espère? Sinon, tu découvriras un autre contenu en appuyant simultanément sur les touches $\mathrm{Ctrl}$ et $\mathrm{W}^{5}$ !

Lelio anticipe avec humour une possible réception fausse de son article par les jeunes lecteurs :

J'admire Charles Baudelaire. Non, ce n'est pas le quatrième orphelin Baudelaire, rendormez-vous. Baudelaire est un poète français du $\mathrm{XIX}^{\mathrm{e}}$ siècle $^{6}$.

Le rédacteur du blog fait allusion aux Désastreuses aventures des orphelins Baudelaire, série romanesque de Lemony Snicket adaptée au cinéma en 2004. Le titre original, A Series of Unfortunate Events, ne mentionne pas le nom «Baudelaire », alors que le titre français favorise une confusion désormais bien établie. D'autres, tel Minotaure, construisent leur blog à la manière d'une anthologie littéraire organisée par auteurs. Au-delà des illustrations choisies - ce sont parfois des photographies personnelles -, le contexte de la fenêtre de navigation lui-même souligne constamment le travail de l'éditeur du blog. Le don du patrimoine est ainsi signé par le médiateur. Contrairement à l'exemple du site « Exercices de styles », analysé par Emmanuël Souchier ${ }^{7}$, ce cas d'anthologie personnelle témoigne d'une conscience du médium chez le rédacteur de blog, qui construit son identité

5. Khazard, « Charles Baudelaire - LXXIv - La cloche fêlé », Le blog de Khazard, 23 janvier 2009, http://khazard.over-blog.fr/article-27105135.html, consulté le 14 avril 2010.

6. Lelio, « Il vaut mieux gâcher sa jeunesse que de n'en rien faire du tout [Georges Courteline] », 24 juin 2009, Lelio Rocks, http://lelio-rocks.over-blog.com/article-33147523.html, consulté le 14 avril 2010.

7. Emmanuël Souchier, « L'exercice de style éditorial. Avatars et réception d'une œuvre à travers l'histoire, des manuscrits à Internet... », Communication \& Langages, ${ }^{\circ} 135$, avril 2003, p. 45-72. 
d'internaute sur sa capacité à mettre en valeur le texte. Présenter au lecteur un poème entièrement copié, c'est offrir un texte qu'on ne possède certes pas, mais s'octroyer en même temps le titre de médiateur. Or, moins la copie représente de travail, plus elle a de chances d'apporter satisfaction au lecteur : le copier-coller simple depuis un site de référence assure l'exactitude du texte, alors que le choix de polices ou de couleurs inhabituelles pour le texte expose au risque de déplaire, et que la copie depuis une édition imprimée peut introduire des erreurs - dont certaines sont plus probablement des faux tout à fait volontaires, comme les articles « Une citation méconnue ${ }^{8}$ » ou encore « Une charogne* ${ }^{*}$ », qui présente en prose un poème versifié. Plus l'intervention sur le texte est grande, plus le risque d'erreur augmente, et moins le consensus est assuré : l'appropriation reste donc essentiellement symbolique, mais le collage du texte constitue bien une pratique rationnelle. Le rédacteur du blog s'autorise de la transmission patrimoniale, aussi redondante soit-elle, pour le plaisir de la collection. Il s'approprie le texte en invitant son lecteur à le faire à son tour, et constitue par là une bibliothèque virtuelle et éclectique qui lui permet de définir son identité sur le réseau.

\section{Une technique de présentation de soi}

Bien que cette fonction du collage relève par définition de l'implicite, la référence au classique littéraire participe à la valorisation du blog et de son auteur. Dans La Distinction, Pierre Bourdieu décrit la pratique de la citation littéraire comme une « sorte de sommation à comparaître au titre de défenseur et de témoin qui est adressée à un auteur du passé sur la base d'une solidarité sociale déguisée en solidarité intellectuelle ${ }^{10} \gg$. On voit bien comment cette solidarité, réelle ou imaginaire, participe à la construction de l'ethos du rédacteur : la citation vaut pour preuve d'un capital culturel suffisant et d'un goût littéraire classique, dont l'exhibition a d'autant plus d'importance que ces qualifications ne sont pas toujours explicitées par le contexte du blog amateur. Particulièrement efficace pour la création d'un consensus culturel entre le rédacteur et son lecteur, la citation d'un poème de Baudelaire suscite de plus des commentaires unanimement approbateurs ; devant l'autorité de la référence, le lecteur, s'il s'exprime, ne peut que se dire conquis :

Merci, c'est très beau. J'ai relu La Fanfarlo aujourd'hui, et j'avais envie d'entendre du Baudelaire. Le reste du site à [sic] l'air appétissant aussi ${ }^{11}$.

8. Hadrien, «Une citation méconnue », $H$ 's, 30 janvier 2009, http://hooblog.wordpress. com/2009/01/30/une-citation-meconnue, consulté le 14 avril 2010. L'auteur du blog reconnaît avoir fait un faux dans un commentaire.

9. Allantverslendroit, « Une charogne*», Allant vers l'endroit, 2 décembre 2008, http://allantverslendroit.wordpress.com/2008/02/12/une-charogne/, consulté le 2 août 2009, blog fermé. 10. Pierre Bourdieu, La Distinction, Paris, Éditions de Minuit, 1979, p. 79.

11. Ulrich, commentaire posté le 25 janvier 2008, sur le blog Minotaure, http://minotaure. 
Les légendes ou les introductions accompagnant le texte sont d'ailleurs souvent construites sur un même modèle superlatif : Baudelaire est « l'un des plus grands poètes français », ou « le premier grand poète "moderne" ». Alors que la mise en question de la valeur du texte est rare, l'éloge est sans appel :

Baudelaire (1821-1867) est sans conteste mon poète préféré. [...] Baudelaire possède un style indémodable, très moderne pour l'époque. Jugez vous même $[$ sic $]:)^{12}$

Le collage du poème ne fait que peu de place à la discussion, et correspond plutôt à une mise en scène de la lecture faite par le rédacteur du blog: dans certains cas, il ne s'agit pas même de donner à lire. Quand l'article ne concerne pas Baudelaire lui-même, le poème intervient ainsi de manière accessoire pour exprimer un goût au détour d'un sujet quotidien, ce qui lui donne un statut mixte, entre le texte et le symbole ${ }^{13}$. Notons qu'au contraire de ce qu'Yves Jeanneret remarquait pour le cas de Romain Rolland, le goût ici manifesté ne paraît ni « impérieux » ni « subjectif ${ }^{14} »$ : déjà validé par la légitimité scolaire de l'œuvre, le choix de Baudelaire peut difficilement paraître original, et le consensus semble aisé. C'est pourquoi certains rédacteurs de blogs se distinguent par le choix d'un poème rarement commenté qui rend compte de la curiosité du copiste. Ainsi « L'Albatros », poème le plus souvent copié, n'a-t-il pas la même valeur dans ce cadre que «L'Irréparable » ou « Fransiscae meae laudes », plus rares sur le réseau. Au même titre que l'évocation encyclopédique, le collage du texte distingue le rédacteur de blog par l'exhibition d'une culture savante mais consensuelle, qui favorise l'adhésion du lecteur à un ethos et à une posture, ce dont les autres articles peuvent bénéficier.

\section{Un déplacement du « je » autobiographique : le lyrisme usurpé?}

Troisième fonction remarquable de la copie du poème, la substitution de la voix du poète à la voix du rédacteur engage un tour de passe-passe complexe. Dans un premier temps, la reprise du poème lyrique par des rédacteurs de blogs peut apparaître comme une preuve de l'universalité des sentiments exprimés par le poète. Cependant, moins de la moitié des pages étudiées concernent Baudelaire lui-même. C'est pourquoi le fait de faire

centerblog.net/1048912-CHARLES-BAUDELAIRE\#i, consulté le 2 août 2009, désormais inaccessible.

12. Méta, « Charles Baudelaire : Les Fleurs du Mal », My Blog-life, http://blogolife.wordpress. com/2009/04/02/charles-baudelaire-les-fleurs-du-mal/, consulté le 14 avril 2010.

13. Emmanuël Souchier montre la tendance à une « iconisation » du texte par les pratiques d'écriture en réseau, dans « L'exercice de style éditorial [...]», op. cit.

14. Yves Jeanneret, « Une conscience européenne sur la toile planétaire : Romain Rolland. net », Communication \& Langages, $\mathrm{n}^{\circ} 135,2003$, p. 30. 
place au « je » du grand auteur lyrique ne peut pas être interprété uniquement comme un hommage : il ne s'agit pas de taire le « je » du rédacteur copiste, mais de le faire passer provisoirement au second plan. Dans la mesure où le contexte d'écriture du poème occupe peu d'espace dans les articles étudiés, la citation abstrait le texte de son origine. Susan Kovacs, dans un article sur la pratique de l'anthologie poétique, décrit cette abstraction d'une manière qui pourrait bien expliquer le phénomène qui nous intéresse : «le statut des poèmes est donc à la fois celui d'un monument et celui d'un énoncé anonyme, réutilisable, virtuel ${ }^{15}$. » Replacé dans le contexte du blog, on voit bien comment cet énoncé anonyme peut être réinvesti par la voix du copiste. Dans le cas limite d'un blog qui ne mentionne pas l'auteur du poème, la citation s'apparente à du plagiat, volontaire ou involontaire : «Hymne à la Beauté » est ainsi rebaptisé « Prière à Lilith, Mère Des Démons ${ }^{16}$ ", et signé par la rédactrice selon un processus automatique propre à la plateforme. Tout se passe comme si le lyrisme impersonnel de Baudelaire et l'appartenance du texte au domaine public autorisaient une appropriation en forme de re-personnalisation, comme si, à mesure que le contexte du poème devenait moins intelligible, il était possible de réinvestir la place laissée vide par l'auteur disparu. Ainsi un autre blog cite-t-il les paroles d'un morceau de Damien Saez très librement inspiré des Femmes damnées ${ }^{17}$. Mêlant les discours des deux femmes de l'œuvre initiale en un seul, les paroles de ce poème recomposé font abstraction du sujet original, l'amour lesbien : elles sont chantées par un homme (Damien Saez) pour une femme. Au-delà de ces deux cas extrêmes, nombreux sont les blogs sur lesquels le texte en morceaux ${ }^{18}$ sert de médium au rédacteur qui, privilégiant l'approche éthique du texte sur l'approche esthétique, trouve dans les mots de Baudelaire de quoi exprimer son propre sentiment. Si le « je » du rédacteur s'efface temporairement, c'est donc pour favoriser l'adhésion de son lecteur : le sentiment exprimé, énoncé par Baudelaire de manière idéale, accède à l'universel.

Le collage du poème met donc involontairement le médium au premier plan : celui-ci prend d'autant plus d'importance que le texte ne recèle pas d'inattendu, puisqu'il est déjà écrit. Cependant, les contradictions du médium sont en même temps mises en lumière : malgré la prédominance

15. Susan Kovacs, « Formes de médiation du texte littéraire : usages de l'anthologie imprimée au XVI ${ }^{\mathrm{e}}$ siècle », Communication \& Langages, $\mathrm{n}^{\circ}$ 129, septembre 2001, p. 110-126.

16. Morticia, «Prière à Lilith, Mère Des Démons », morticia57070, http://morticia57070. centerblog.net/10.html, consulté le $1^{\text {er }}$ septembre 2008, dernière mise à jour le 2 janvier 2008 , n'est plus accessible aujourd'hui.

17. Thierry Ledru, «Baudelaire, Damien Saez, Gregory Colbert », dans l'espace «blog » du Site de Thierry Ledru, http://jarwal.e-monsite.com/blog,baudelaire-damien-saez-gregorycolbert,139835.html, consulté le 2 août 2009.

18. Comme le note Pierre Assouline dans son article « Baudelaire en morceaux », l'ensemble de l'œuvre pourrait être recomposé à partir des morceaux baudelairiens éparpillés entre les sites. Pierre Assouline, « Baudelaire en morceaux », La République des livres, http://passouline.blog.lemonde.fr/2009/04/19/baudelaire-en-morceaux, consulté le 2 août 2009. 
d'une écriture à la première personne, le rédacteur de blog a conscience de pratiquer une écriture publique et la citation littéraire, technique de valorisation de l'image de soi, est de manière indirecte un signe de la prise en compte du public. D'un autre côté, cette pratique remplit aussi une fonction qu'Emmanuël Souchier ${ }^{19}$ qualifie d' " hédoniste » : la copie du texte s'accompagne, sinon d'une relecture, du souvenir d'une lecture plaisante; elle est à soi-même sa propre fin. La question que posait Yves Jeanneret dans son article sur les sites faisant référence à Romain Rolland trouve donc une réponse simple pour le cas des blogs collant le texte de Baudelaire. Dans son exploration d'un « vide du réseau ${ }^{20} »$, Yves Jeanneret pouvait légitimement se demander si les écrits d'écran « sont [...] réellement le lieu d'une contre culture » ou s'ils « apportent leur concours au discours dominant 》 dans la diffusion des textes et des références. Dans le cas des blogs, et dans le cas de Baudelaire, qui correspond, lui, à un « plein » du réseau, le discours dominant n'est pas mis en question, et le texte, bien présent, ne fait que rarement l'objet d'une relecture. Citer Baudelaire, c'est donc le mettre au centre de la page mais non au centre du discours : si le collage du texte se présente comme une pratique de transmission, le texte transmis est souvent déjà connu, et la citation littéraire constitue plutôt un moyen d'interaction avec le lecteur qui permet au rédacteur, ici copiste, de redéfinir à son gré sa propre identité virtuelle.

Mathilde Labbé

Université de Paris-Sorbonne

Centre de recherche sur la littérature française $d u X I X X^{e}$ siècle

19. Emmanuël Souchier, «L'exercice de style éditorial... », op. cit., p. 72.

20. Yves Jeanneret, op. cit., p. 29. 